\title{
MicroRNA-137 suppresses the proliferation, migration and invasion of cholangiocarcinoma cells by targeting WNT2B
}

\author{
TENGXIANG CHEN ${ }^{1,2}$, SHAN LEI $^{1,2}$, ZHIRUI ZENG $^{1,2}$, SHUTAO PAN $^{3}$, JINJUAN ZHANG $^{1,2}$,

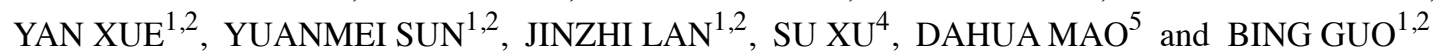 \\ ${ }^{1}$ Guizhou Provincial Key Laboratory of Pathogenesis and Drug Research on Common Chronic Diseases; ${ }^{2}$ Department of \\ Physiology, School of Basic Medical Sciences, Guizhou Medical University, Guiyang, Guizhou 550009; ${ }^{3}$ Department of \\ Biliary-Pancreatic Surgery, Affiliated Tongji Hospital, Tongji Medical College, Huazhong University of Science and \\ Technology, Wuhan, Hubei 430060; ${ }^{4}$ Department of Pathology, Affiliated Hospital of Guizhou Medical University; \\ ${ }^{5}$ Department of Breast Surgery, Wudang Affiliated Hospital, School of Clinical Medical Science, Guiyang, \\ Guizhou 550009, P.R. China
}

Received June 18, 2019; Accepted December 17, 2019

DOI: $10.3892 /$ ijmm.2020.4474

\begin{abstract}
It is widely known that abnormal regulation of microRNAs (miRNAs/miRs) may contribute to the occurrence or development of tumors. The objective of the present study was to elucidate the function and underlying mechanism of miR-137 in the progression of cholangiocarcinoma (CCA). The expression levels of miR-137 in CCA tissues and cell lines were measured using reverse transcription-quantitative PCR. The role of miR-137 in the proliferation of CCA cells was assessed using the Cell Counting Kit- 8 assay, colony formation assay and cell cycle distribution analysis, while its effects on the migration and invasion of CCA cells were evaluated using Transwell assays. The function of miR-137 on CCA growth in vivo was also investigated using a xenograft mouse model. Furthermore, the association between miR-137 and Wnt family member 2B (WNT2B) was analyzed using bioinformatics, double luciferase assay and western blotting. It was verified that the expression of miR-137 was low in CCA tissues and cell lines, whereas increased expression of miR-137 significantly suppressed cell proliferation, decreased colony formation ability and induced G1 phase arrest. miR-137 overexpression suppressed the migration and invasion ability of TFK-1 and HuCCT1 cells. Furthermore, the results of the xenograft mouse model assays revealed that miR-137 overexpression decreased tumor growth in vivo. The results of bioinformatics analysis and dual luciferase reporter assays demonstrated that WNT2B is directly regulated by miR-137.
\end{abstract}

Correspondence to: Professor Tengxiang Chen, Department of Physiology, School of Basic Medical Sciences, Guizhou Medical University, 1 Dongqing Road, Guiyang, Guizhou 550009, P.R. China E-mail: txch@gmc.edu.cn

Key words: cholangiocarcinoma, microRNA-137, proliferation, Wnt family member $2 \mathrm{~B}$, invasion, metastasis
The expression of WNT2B and Wnt-pathway-related proteins was decreased when miR-137 was overexpressed. Restoring the expression of WNT2B notably reversed the inhibitory effect of miR-137 on CCA cells. Therefore, the findings of the present study demonstrated that miR-137 acts as a suppressor in CCA and inhibits CCA cell proliferation, migration and invasion through suppressing the expression of WNT2B.

\section{Introduction}

Cholangiocarcinoma (CCA) is known as one of the most aggressive malignancies worldwide. CCA is responsible for $5-15 \%$ of all primary liver cancer cases and is the second most frequent type of liver cancer (1). CCA is a highly invasive malignancy, exhibiting early distal organ and lymph node metastasis and resistance to chemotherapy $(2,3)$. In recent years, the morbidity and mortality of CCA have increased worldwide (4). Therefore, it is crucial to elucidate the mechanism underlying the occurrence and progression of CCA in order to uncover new cancer treatment targets.

MicroRNAs (miRNAs/miRs), a class of non-coding RNA molecules consisting of 18-25 nucleotides, have been verified to regulate the translation of various target genes and are involved in cancer occurrence, metastasis, progression and recurrence $(5,6)$. In CCA, certain miRNAs have been previously demonstrated to exert stimulatory/suppressive effects on cancer progression (7). For example, miR-383 was found to act as a tumor suppressor in CCA and inhibits tumor development through decreasing the expression of interferon regulatory factor 1 (8). Through inhibiting aldolase A translation, miR-122-5p decreased bile duct carcinoma cell proliferation and promoted cell apoptosis (9). By regulating autophagy-related cell death, miR-124 was shown to act as tumor suppressor in CCA (10).

The function and underlying mechanism of action of miR-137 have been investigated in various types of cancer (11-14). A study on non-small cell lung cancer revealed that miR-137 may decrease tumor growth and sensitize cancer 
cells to chemotherapy via regulating AKT2 (15). Similarly, in colon cancer, patients with low expression levels of miR-137 have adverse clinical prognosis $(16,17)$. However, the biological function and molecular mechanism of action of miR-137 in CCA remain elusive.

The aim of the present study was to investigate the expression of miR-137 in CCA tissues and cell lines, and elucidate whether its effects on the proliferation, migration and invasion ability of CCA cells were mediated via regulation of Wnt family member 2B (WNT2B), in order to determine whether miR-137 and its target gene, WNT2B, may be novel biomarkers for the diagnosis and clinical treatment of CCA.

\section{Materials and methods}

Tissue samples. A total of 29 patients (mean age, 52.3 \pm 7.2 years; range, 44-65 years; 13 men and 16 women) from the Affiliated Hospital of Guizhou Medical University (Guizhou, China) were enrolled in the present study between January 2018 and March 2019. The inclusion criteria were as follows: i) The tissues were obtained during surgery and diagnosed as CCA by two pathologists; ii) patients diagnosed and treated for the first time; and iii) patients consented to participate. The exclusion criteria were as follows: i) Cases complicated with other malignancies; ii) presence of other systemic diseases; iii) patients receiving treatment prior to admission; and iv) patients and/or their families refusing to participate. All 29 patients provided CCA tissue samples, while adjacent normal tissues were also obtained from 20 of the patients. The Human Trial Ethics Committee of Guizhou Medical University approved the study. Written informed consent was obtained from the patients who provided the specimens. The present study was performed in accordance with the principles outlined in the Declaration of Helsinki. All samples were kept in liquid nitrogen until used.

Cell culture and lentivirus infection. The four CCA cell lines (TFK-1, HuCCT1, RBE and QBC939) and human intrahepatic biliary epithelial cells (HIBEpiC) were purchased from the Cell Bank of Chinese Academy of Sciences. All cell lines used in the present study were cultured in RPMI-1640 medium (Invitrogen; Thermo Fisher Scientific, Inc.) containing 10\% fetal bovine serum (FBS; Gibco; Thermo Fisher Scientific, Inc.). Human miR-137 overexpression lentivirus (LV-miR-137) and corresponding negative control (NC) lentivirus were obtained from Shanghai GeneChem Co., Ltd. The whole infection procedure was performed based on the protocol provided by the manufacturer (MOI=10 for both TFK-1 and HuCCT1 cells). The infection efficacy was evaluated by a fluorescence microscope (magnification, $\mathrm{x} 200$ ) and reverse transcription-quantitative PCR (RT-qPCR).

$R T$ - $q P C R$. According to the protocol provided by the manufacturer, total RNA was extracted from cells or tissues using TRIzol (Yeasen). cDNA was obtained via RT of total RNA using Prime Script RT Master Mix (Yeasen) and a miRNA First Strand cDNA Synthesis (Stem-loop Method) kit (Yeasen) for mRNA and miRNA, respectively. The protocol for RT of mRNA and miRNA was $37^{\circ} \mathrm{C}$ for $15 \mathrm{~min}, 85^{\circ} \mathrm{C}$ for $30 \mathrm{sec}$ and $4^{\circ} \mathrm{C}$ for $5 \mathrm{~min}$. The expression levels were measured using
SYBR reagent (Yeasan), while GAPDH and U6 were employed as the reference gene for mRNAs and miRNA, respectively. Relative expression levels of genes were analyzed using the $2^{-\Delta \Delta \mathrm{Cq}}$ method (18). The primers used were as follows: WNT2B: Forward, 5'-GGGGCACGAGTGATCTGTG-3' and reverse, 5'-GCATGATGTCTGGGTAACGCT-3'; c-Myc: Forward, 5'-GGCTCCTGGCAAAAGGTCA-3' and reverse, 5'-CTG CGTAGTTGTGCTGATGT-3'; N-cadherin: Forward, 5'-TCA GGCGTCTGTAGAGGCTT-3' and reverse, 5'-ATGCACATC CTTCGATAAGACTG-3'; vimentin: Forward, 5'-GACGCC ATCAACACCGAGTT-3' and reverse, 5'-CTTTGTCGTTGG TTAGCTGGT-3'; cyclin D1: Forward, 5'-GCTGCGAAGTGG AAACCATC-3' and reverse, 5'-CCTCCTTCTGCACACATT TGAA-3'; cyclin-dependent kinase (CDK)2: Forward, 5'-CCA GGAGTTACTTCTATGCCTGA-3' and reverse, 5'-TTCATC CAGGGGAGGTACAAC-3'; GAPDH: Forward, 5'-GGA GCGAGATCCCTCCAAAAT-3' and reverse, 5'-GGCTGT TGTCATACTTCTCATGG-3'; U6: Forward, 5'-TCGCTT CGGCAGCACATATACT-3' and reverse, 5'-ACGCTTCAC GAATTTGCGTGTC-3'. The thermocycling conditions in the present study were: $95^{\circ} \mathrm{C}$ for $5 \mathrm{~min}, 40$ cycles of $95^{\circ} \mathrm{C}$ for $30 \mathrm{sec}$, annealing at $60^{\circ} \mathrm{C}$ for $30 \mathrm{sec}$ and a final elongation step at $72^{\circ} \mathrm{C}$ for $30 \mathrm{sec}$.

Cell Counting Kit-8 (CCK-8) assay. TFK-1 and HuCCT1 cells at a density of $2 \times 10^{3}$ cells/well were cultured in 96-well plates (Wuhan Boster Biological Technology, Ltd.) with $100 \mathrm{ml}$ RPMI-1640 following infection with LV-miR-137 and NC lentivirus. After culturing for 24, 48, 72 and $96 \mathrm{~h}$, a total of $10 \mu \mathrm{l} \mathrm{CCK}-8$ reagent (Wuhan Boster Biological Technology, Ltd.) was added into each well, according to the manufacturer's protocol. After culturing for $2 \mathrm{~h}$, cell proliferation ability was measured by a spectrophotometer (Bio-Rad Laboratories, Inc.) at an optical density (OD) of $450 \mathrm{~nm}$.

Colony formation assay. Cells were seeded onto 6-well plates (Wuhan Boster Biological Technology, Ltd.) at a density of $2 \times 10^{3}$ and cultured in RPMI-1640 medium containing $10 \%$ FBS. After 2 weeks of incubation, the colonies were fixed for $30 \mathrm{~min}$ at room temperature using $4 \%$ paraformaldehyde. The number of colonies was counted by naked eye after staining with $0.1 \%$ crystal violet solution for $20 \mathrm{~min}$ at room temperature.

Cell cycle distribution analysis. TFK-1 and HuCCT1 cells were resuspended in serum free RMPI-1640 medium and seeded into 6 -well plates at a density of $2 \times 10^{5}$ cells per well. Then, cells were starved for $24 \mathrm{~h}$ using RPMI-1640 without FBS for cell cycle synchronization. After cell cycle synchronization, cells were treated with RMPI-1640 medium containing $10 \%$ FBS and allowed to proliferate for $48 \mathrm{~h}$. Then, the cells were harvested and fixed using $75 \%$ ethanol (Servicebio) for $24 \mathrm{~h}$ at $-20^{\circ} \mathrm{C}$. After washing with PBS, the cells were treated with $5 \mu 1$ propidium iodide (Invitrogen; Thermo Fisher Scientific, Inc.) and $1 \mathrm{X}$ binding buffer at room temperature for $30 \mathrm{~min}$. Finally, the cell cycle distribution was examined using a BD FACScan ${ }^{\mathrm{TM}}$ flow cytometer (BD Biosciences; Becton, Dickinson and Company; version no. 343039) and FlowJo software was used to analyze the results (version 7.4.1; FlowJo LLC). 
Migration and invasion Transwell assays. TFK-1 and HuCCT1 cells $\left(2 \times 10^{5}\right)$ were resuspended in $200 \mu 1$ RPMI-1640 medium without FBS and added into the upper Transwell chamber (EMD Millipore) with or without Matrigel (Invitrogen; Thermo Fisher Scientific, Inc.), while $700 \mu 1$ medium containing 10\% serum was placed into the lower Transwell chamber. After culturing cells for $24 \mathrm{~h}$, cells not migrating or invading through the membranes $(0.8 \mu \mathrm{m})$ were removed and cells crossing the membranes were fixed using $4 \%$ paraformaldehyde (Servicebio) for $20 \mathrm{~min}$ at room temperature and stained with $1 \%$ crystal violet solution (Servicebio) for $30 \mathrm{~min}$ at room temperature. A total of five different fields were photographed using an inverted optical microscope (Nikon Corporation; magnification, $\mathrm{x} 40$ ) and the cells were counted.

Animal experiments. Female BALB/c nude mice $(\mathrm{n}=12)$, aged 4 weeks and weighing $\sim 17 \mathrm{~g}$, were purchased from the Beijing Vital River Laboratory Animal Technology Co., Ltd. The mice were housed under specific pathogen-free conditions at $25^{\circ} \mathrm{C}$ with a 12-h light/dark cycle and free access to food and water. First, the miR-137-overexpressing HuCCT1 cell line and normal control were constructed. Next, a total of $1 \times 10^{7}$ cells suspended in PBS were injected into the subcutaneous tissues of the right upper flank in mice ( $n=6$ per group). The animal health and behavior were monitored daily, and the volume of tumor tissue was measured weekly and calculated using the following formula: Volume $\left(\mathrm{mm}^{3}\right)=\left(\right.$ length $\mathrm{x}$ width $\left.{ }^{2}\right) / 2$. When the maximum length of any tumor reached $15 \mathrm{~mm}$ or the volume of any tumor reached $800 \mathrm{~mm}^{3}$, the experiment was terminated. All mice ( $\mathrm{n}=12$; no mice died during the experiment) were sacrificed using cervical dislocation and death was verified by the absence of a heartbeat and the onset of rigor mortis. Subsequently, the tumor tissues were isolated and weighed. All animal experiments were approved by the Ethics Committee of Guizhou Medical University.

Immunohistochemistry. After fixation using 4\% paraformaldehyde for $24 \mathrm{~h}$ at room temperature, dehydration and embedding in paraffin (Servicebio), the tissues were cut into $4-\mu \mathrm{m}$ sections. The specimens were then deparaffinized using xylene and rehydrated through graded alcohols. After restoration with sodium citrate and blocking using $\mathrm{H}_{2} \mathrm{O}_{2}$ and bovine serum albumin (Servicebio), the specimens were incubated with the primary antibodies (both 1:400), including Ki-67 (cat. no. 27309-1-AP) and proliferating cell nuclear antigen (PCNA; cat. no. 10205-2-AP; ProteinTech Group, Inc.) for $12 \mathrm{~h}$ at $4^{\circ} \mathrm{C}$. Subsequently, the sections were immunohistochemically stained with horseradish peroxidase (HRP)-conjugated goat anti-rabbit secondary antibodies (cat. no. G1213; Servicebio) for $2 \mathrm{~h}$ at room temperature. Following incubation with the Cell and Tissue Staining HRP-DAB kit for $5 \mathrm{~min}$ at room temperature (Servicebio), an orthophotomicroscope (Nikon Corporation; magnification, $\mathrm{x} 400$ ) was used to capture images.

miRNA target prediction. Potential targets of miR-137 was predicted using online website TargetScan (http://www. targetscan.org/; version no. 7.2). Target genes with an absolute value of cumulative weighted context ++ score $>0.2$ were considered credible. Credible target genes of miR-137 were imported to the online tool Database for Annotation, Visualization and Integrated Discovery (https://david.ncifcrf. gov/; version no. 6.8) to perform Kyoto Encyclopedia of Genes and Genomes analysis. $\mathrm{P}<0.05$ was set as the cutoff to consider significant enrichment. The binding site of vital target genes in significantly enriched pathways was shown and the association between miR-137 and the vital target gene was further examined.

Dual luciferase reporter assays. In order to verify whether miR-137 binds to WNT2B and regulates its expression, a dual luciferase reporter assay was performed. The wild-type (Wt) and mutant (Mut) type 3'-untranslated regions (UTRs) of WNT2B, which contain predicted binding sites for miR-137, were synthesized and subcloned into the psiCHECK-2 luciferase reporter vector (Promega Corporation). After culturing for $24 \mathrm{~h}, \mathrm{TFK}-1$ and HuCCT1 cells were co-transfected with Wt/Mut type WNT2B luciferase reporter vector and miR-137 mimic (sequence: 5'-UUAUUGCUUAAGAAU ACGCGUAG-3'). miR-137 NC (sequence: 5'-UCACAACCU CCUAGAAAGAGUAGA-3'). Finally, the Dual Luciferase Reporter Assay System (Promega Corporation) was employed to measure the luciferase activities, and the activities were normalized with Renilla luciferase activity. Lipofectamine ${ }^{\circledR}$ 2000 (Invitrogen; Thermo Fisher Scientific, Inc.) was used for transient transfection, and the duration between transfection and activity measurement was $24 \mathrm{~h}$.

Western blotting. Cells were lysed using a RIPA buffer (Wuhan Boster Biological Technology, Ltd.) containing protease inhibitor cocktail (Boster Biological Technology) and PMSF (Wuhan Boster Biological Technology, Co., Ltd.). Following centrifugation $(8,000 \mathrm{x} \mathrm{g} / 15 \mathrm{~min})$ at $4^{\circ} \mathrm{C}$, proteins were collected from cellular debris and the bicinchoninic acid method was employed to determine the concentration. Protein samples (30 $\mu \mathrm{g} /$ well) were separated using 10\% SDS-PAGE under $90 \mathrm{~V}$ voltage and transferred onto PVDF membranes (EMD Millipore). After blocking with TBST (0.1\% Tween-20) containing 5\% skimmed milk at room temperature for $2 \mathrm{~h}$, the membranes were then incubated with primary antibodies $(1: 1,000)$ against WNT2B (cat. no. ab50575; Abcam), $\beta$-catenin (cat. no. 51067-2-AP; Proteintech), TCF4 (cat. no. 22337-1-AP; Proteintech), N-cadherin (cat. no. 22018-1-AP; Proteintech), vimentin (cat. no. 10366-1-AP; Proteintech), cyclin D1 (cat. no. 26939-1-AP; Proteintech), CDK2 (cat. no. 10122-1-AP; Proteintech), c-Myc (cat. no. 10828-1-AP; Proteintech) and GAPDH (cat. no. 60004-1-Ig; Proteintech) for $16 \mathrm{~h}$ at $4^{\circ} \mathrm{C}$. After washing with TBST to remove unbound antibodies, the membranes were incubated with the horseradish peroxidase conjugated secondary antibodies goat anti-rabbit (1:3,000; cat. no. SA00001-2, Proteintech) and goat anti-mouse (1:3,000; cat. no. SA00001-1, Proteintech) for $2 \mathrm{~h}$ at room temperature. Finally, the signals were detected by the Photoshop Image Analysis software CS3 (Adobe Systems).

Statistical analyses. All experiments were repeated three times and the data are presented as the mean \pm standard deviation. All statistical analyses in the present study were performed using SPSS 21.0 (IBM Corp.). Comparisons between two groups were conducted using two-tailed Student's t-test, while 

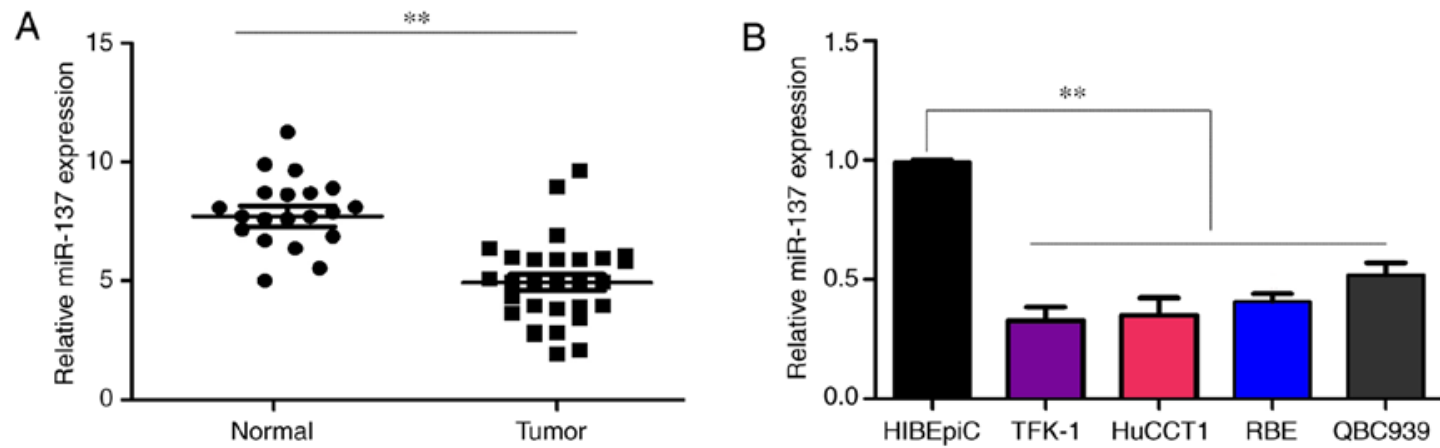

Figure 1. miR-137 is significantly downregulated in cholangiocarcinoma. (A) The expression of miR-137 was detected in 29 cholangiocarcinoma tissues and 20 adjacent non-tumor tissues using reverse transcription-quantitative PCR. (B) The expression of miR-137 in the cholangiocarcinoma cell lines TFK-1, HuCCT1, RBE and QBC939 and HIBEpiCs was detected using reverse transcription-quantitative PCR. ${ }^{* *} \mathrm{P}<0.01$. miR, microRNA; HIBEpiCs, human intrahepatic biliary epithelial cells.

comparisons among multiple groups were performed using one-way analysis of variance combined with LSD-t test. The co-expression relationship between two genes was analyzed using Pearson relationship analysis. $\mathrm{P}<0.05$ was considered to indicate statistically significant differences.

\section{Results}

miR-137 is significantly downregulated in CCA. To determine whether miR-137 was involved in the progression of CCA, its expression in 29 histologically confirmed CCA samples and 20 adjacent non-tumor samples was detected using RT-qPCR. The results demonstrated the expression of miR-137 was significantly decreased in the CCA tissues compared with that in adjacent non-tumor tissues (Fig. 1A). Similarly, it was demonstrated that the expression level of miR-137 was lower in CCA cell lines, including TFK-1, HuCCT1, RBE and QBC939, compared with that in HIBEpiCs (Fig. 1B). Taken together, these results indicate that the loss of expression of miR-137 may be involved in the development of CCA.

miR-137 represses $C C A$ cell proliferation in vitro. First, the CCA cell lines HuCCT1 and TFK-1 were stably infected with miR-137 overexpression (LV-miR-137) and NC lentiviruses. The results revealed that both LV-miR-137 and NC lentiviruses exhibited high infection efficiency in HuCCT1 cells after infection for $24 \mathrm{~h}(>80 \%)$. Similarly, compared with cells infected with NC lentiviruses, the mRNA levels of miR-137 were significantly increased in cells infected with LV-miR-137 lentiviruses (Fig. 2A). CCK-8 assays were used to detect the effect of miR-137 on CCA cell proliferation ability and the results revealed that miR-137 overexpression notably inhibited the proliferation ability of CCA cells (Fig. 2B). Additionally, the results of colony formation assays demonstrated that increased expression of miR-137 inhibited the colony formation ability of CCA cells (Fig. 2C). Moreover, cell cycle distribution analysis revealed that the percentage of HuCCT1 and TFK-1 cells in the G1 phase was significantly increased in the LV-miR-137 group compared with the NC groups, while the number of cells in the $\mathrm{S}$ phase was markedly decreased (Fig. 2D). Furthermore, western blotting was performed to measure the expression levels of CDK2 and cyclin D1, which are involved in regulating cell transition from the G1 to the $\mathrm{S}$ phase. The results of western blotting revealed that the expression of CDK2 and cyclin D1 decreased following infection with miR-137 overexpression lentiviruses (Fig. 2E).

miR-137 represses $C C A$ cell migration and invasion in vitro. The results of the Transwell assays demonstrated that both the migration and invasion abilities in the miR-137 overexpression groups were decreased compared with the control groups (Fig. 3A). Previous studies demonstrated that epithelial-to-mesenchymal transition (EMT) is a crucial process for cancer cell mobility (19-21). To determine whether miR-137 inhibits the mobility of HuCCT1 and TFK-1 cells via affecting the EMT phenotype, western blotting was conducted to detect the protein levels of two key markers of EMT, N-cadherin and vimentin. It was demonstrated that the protein levels of both $\mathrm{N}$-cadherin and vimentin were decreased with miR-137 overexpression (Fig. 3B).

miR-137 significantly inhibits tumor growth in vivo. Having uncovered the vital impact of miR-137 in the biology of CCA cells in vitro, the effect of miR-137 on tumor growth in vivo was next investigated. For this purpose, HuCCT1 cells stably expressing miR-137 or miR-NC were injected into the subcutaneous tissues of nude mice and tumor growth was monitored. The results revealed that the growth rate of tumors derived from miR-137-overexpressing HuCCT1 cells was significantly slower and the formed tumors were significantly smaller compared with those originating from miR-NC cells (Fig. 4A and B). In addition, the weight of the mice decreased more slowly in the miR-137 overexpression group (Fig. 4C). Furthermore, miR-137-overexpressing tumors excised after 5 weeks exhibited markedly decreased levels of the proliferation marker Ki-67 and PCNA proteins compared with miR-NC tumors, as determined by immunohistochemical examination (Fig. 4D).

WNT2B is a key target of miR-137 in CCA. To uncover the molecular mechanism underlying the role of miR-137 in regulating the function of CCA cells, the online bioinformatics tool TargetScan was employed to identify mRNAs containing 3'UTR sequences complementary to miR-137. As the results demonstrated, one of the key pathways in which the credible target genes of miR-137 were enriched was the 
A
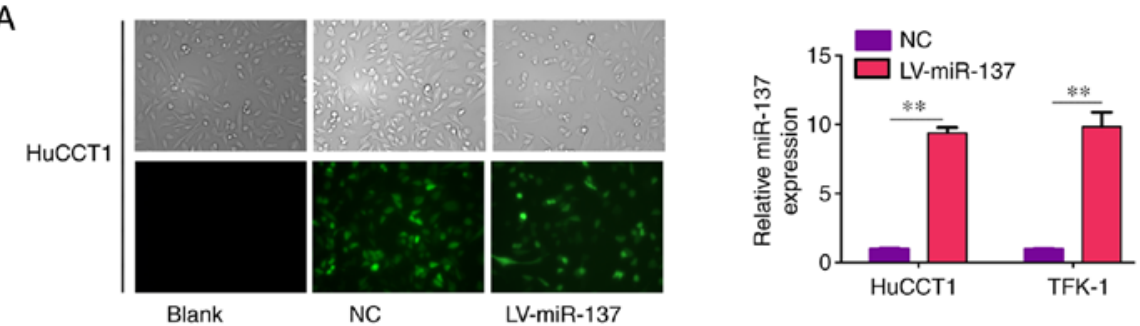

B
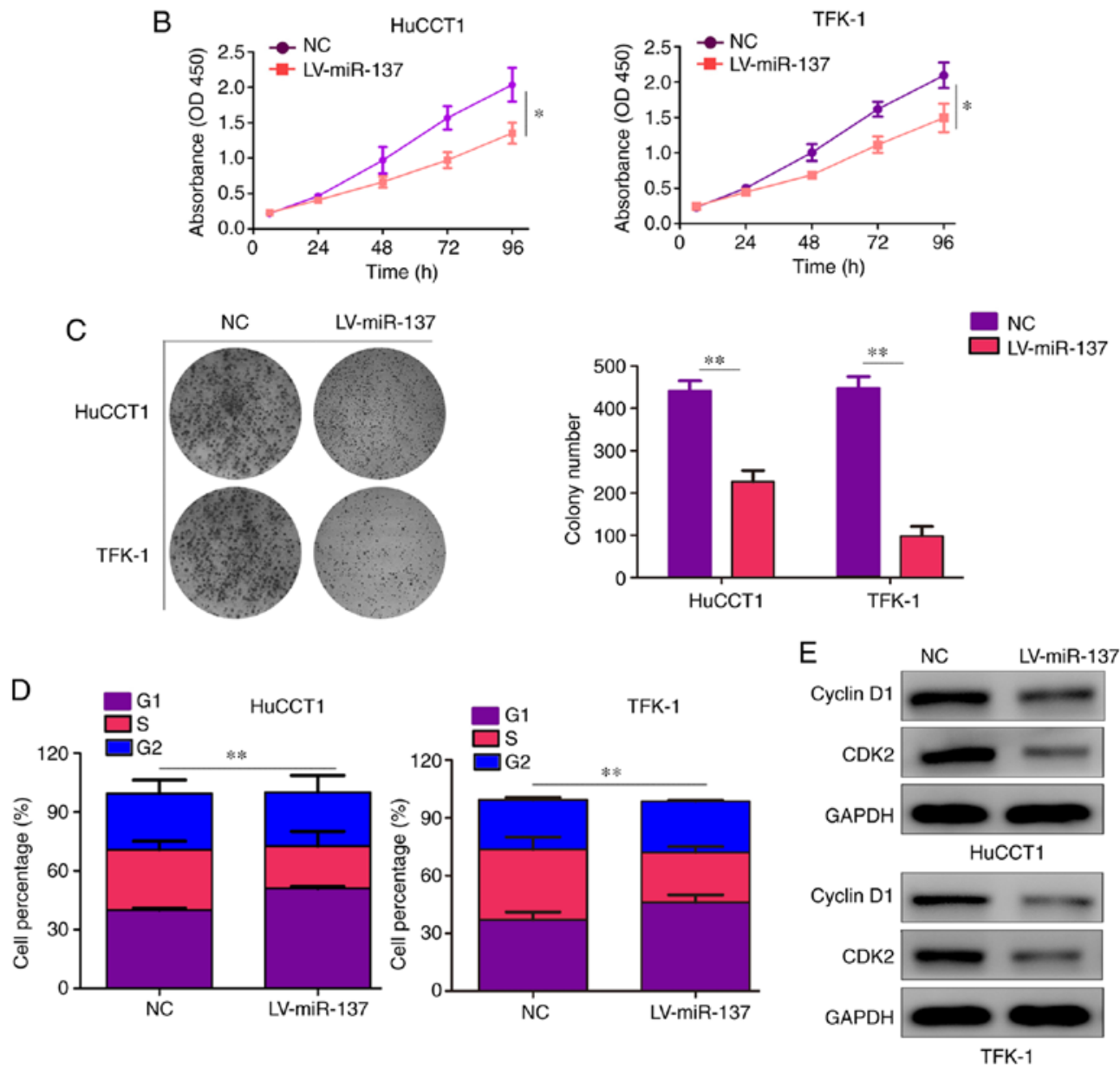

Figure 2. miR-137 represses cholangiocarcinoma cell proliferation in vitro. (A) Fluorescence microscope examination (magnification, x200) and reverse transcription-quantitative PCR were used to detect the infection efficiency of miR-137 overexpression (LV-miR-137) and NC lentiviruses. (B) The effect of miR-137 on cholangiocarcinoma cell proliferation was detected by the Cell Counting Kit-8 assay. (C) The effect of miR-137 on cholangiocarcinoma cell colony formation ability was detected using colony formation assays. (D) Cell cycle distribution was analyzed after miR-137 overexpression in TFK-1 and HuCCT1 cells. (E) Western blotting was used to detect the expression of CDK2 and cyclin D1 in the miR-137 overexpression and normal control groups. GAPDH was used as the loading control. ${ }^{*} \mathrm{P}<0.05,{ }^{* *} \mathrm{P}<0.01$. miR, microRNA; NC, negative control; $\mathrm{LV}$, lentivirus; CDK, cyclin dependent kinase; OD, optical density.

Wnt signaling pathway (Fig. 5A). In addition, the 3'UTR of WNT2B, which plays a key role in the Wnt signaling pathway, contained a putative miR-137-binding site (Fig. 5B). Therefore, WNT2B may be an important target of miR-137. To validate the prediction, the $3^{\prime} \mathrm{UTR}$ of WNT2B, either Wt or Mut, in the putative binding site of miR-137 was cloned into a luciferase reporter vector, which was transfected into TFK-1 and HuCCT1 cells together with miR-137 or miR-NC. The results indicated that co-transfection with miR-137 decreased luciferase activity driven by WNT2B-Wt, but not by WNT2B-Mut (Fig. 5C). Similarly, increased expression of miR-137 decreased the mRNA level of WNT2B in both TFK-1 and HuCCT1 cells (Fig. 5D). Subsequently, correlation analysis proved that the mRNA levels of WNT2B were negatively associated with miR-137 in the 29 human CCA samples (Fig. 5E). Furthermore, the mRNA level of WNT2B was higher in CCA samples and cell lines compared with normal samples (Fig. 5F and G).

miR-137 regulates the Wnt signaling pathway via WNT2B. Based on the fact that WNT2B is a key factor in the Wnt signaling pathway $(22,23)$, whether miR-137 may regulate Wnt signaling pathway via WNT2B was investigated. The results of western blotting demonstrated that miR-137 overexpression decreased the protein level of WNT2B, $\beta$-catenin and TCF4, while ectopic expression of WNT2B was able to reverse this effect induced by miR-137 (Fig. 6A and B). Furthermore, it was observed that both the transcription and translation levels of 
A

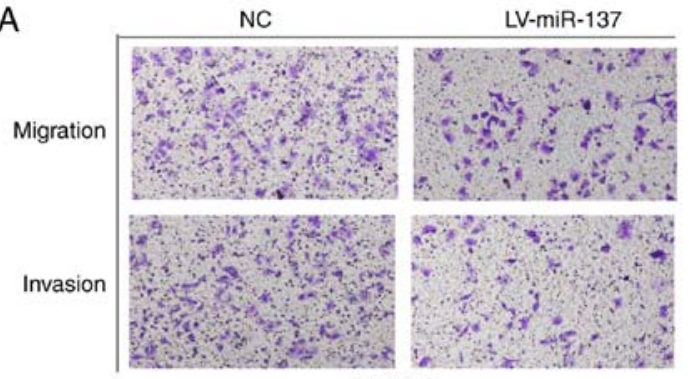

HuCCT1

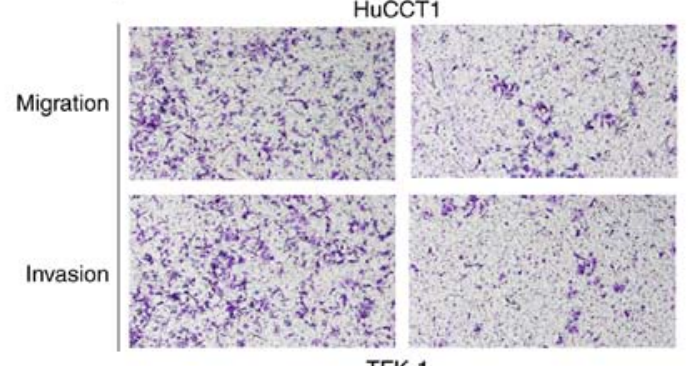

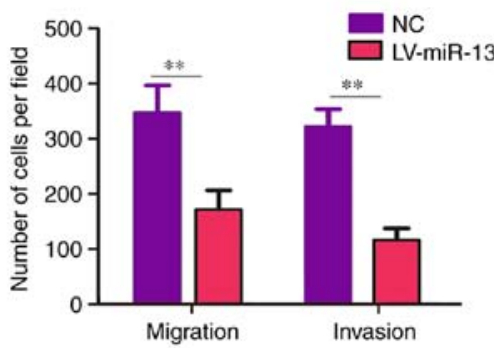

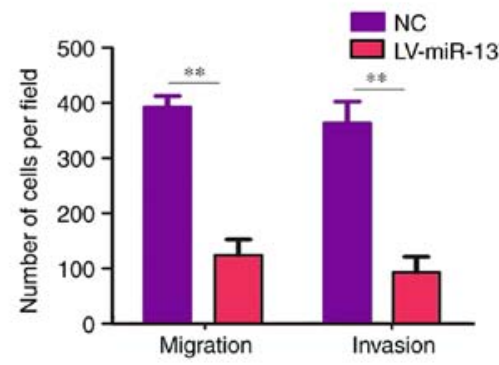

B
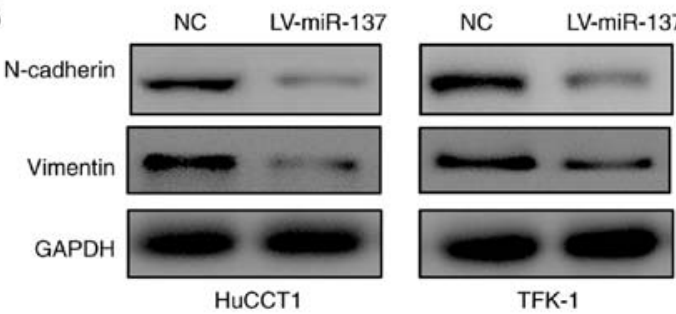

Figure 3. miR-137 represses cholangiocarcinoma cell migration and invasion in vitro. (A) The effect of miR-137 on cholangiocarcinoma cell invasion and migration was examined by Transwell assays. Magnification, $\mathrm{x} 40$. (B) Western blotting was used to detect the expression of N-cadherin and vimentin in the miR-137 overexpression and normal control groups. GAPDH was used as the loading control. ${ }^{* *} \mathrm{P}<0.01$. miR, microRNA; NC, negative control; LV, lentivirus; $\mathrm{N}$, neural.

A
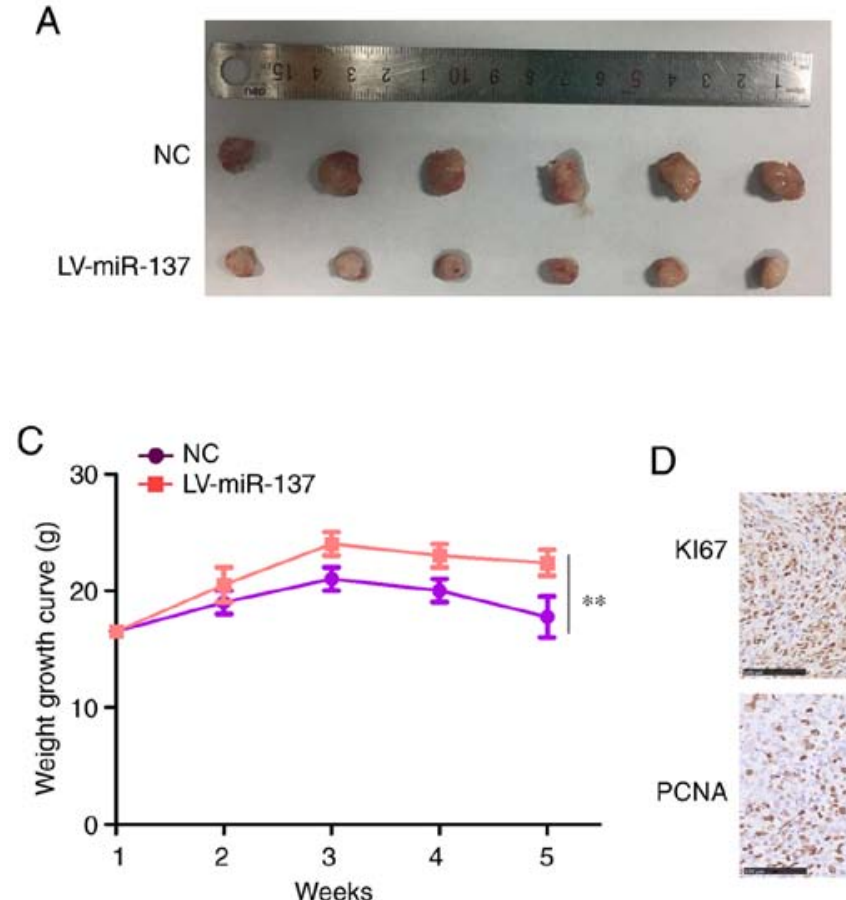

D
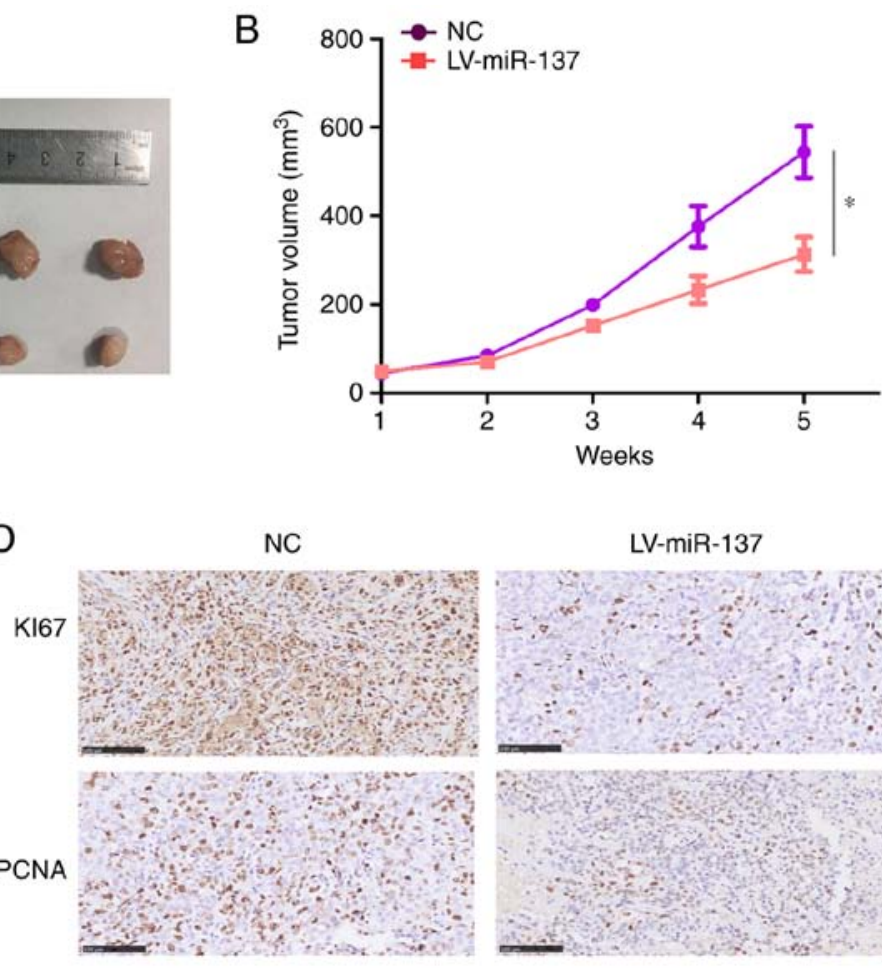

LV-miR-137

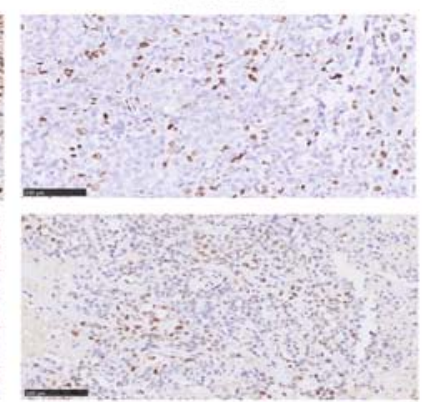

Figure 4. miR-137 inhibits tumor growth in vivo. (A) Representative images of subcutaneous tumors of the miR-137 overexpression and control groups. (B) HuCCT1 cells stably expressing miR-137 or miR-NC were injected into the subcutaneous tissues of nude mice, and tumor growth was monitored over 5 weeks. (C) The weight of the mice in the miR-137 overexpression and miR-NC groups was measured weekly. (D) The expression of Ki-67 and PCNA in miR-137-overexpressing tumors and miR-NC-expressing tumors was detected by immunohistochemistry staining. Scale bars, $100 \mu \mathrm{m}$. ${ }^{*} \mathrm{P}<0.05,{ }^{* *} \mathrm{P}<0.01$. PCNA, proliferating cell nuclear antigen; NC, negative control; LV, lentivirus; miR, microRNA. 

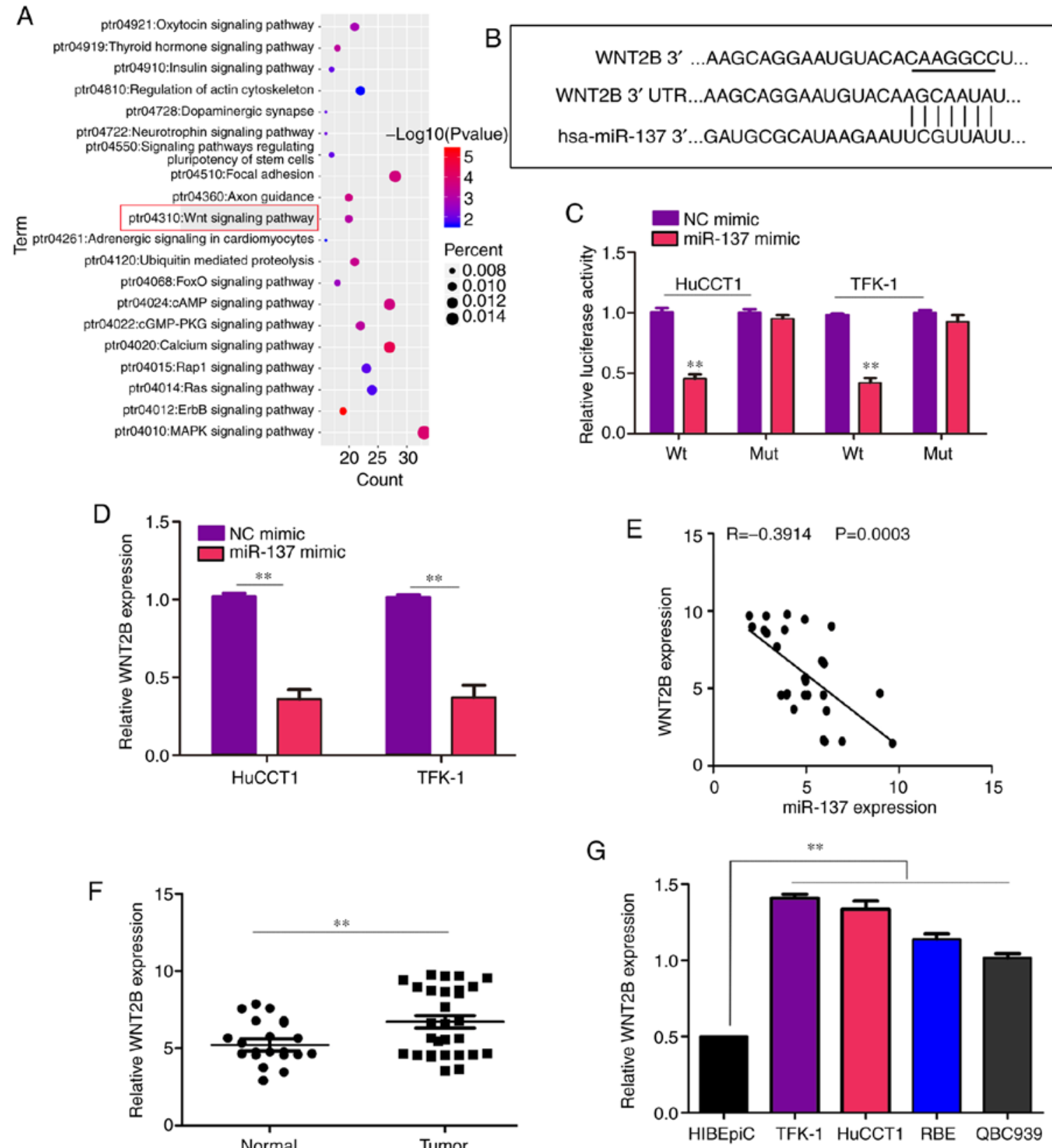

Figure 5. WNT2B is a key target of miR-137 in cholangiocarcinoma. (A) Bubble chart showing the pathways of the miR-137 target genes were enriched in. (B) miR-137 may bind to the 3'-UTR of WNT2B mRNA. The underlined sequence is the mutated site. (C) miR-137 mimics inhibited luciferase activity in cholangiocarcinoma cells, while mutation of the 3'-UTR of WNT2B mRNA abolished the effect of miR-137 mimic on luciferase activity. (D) Overexpression of miR-137 decreased the mRNA expression level of WNT2B in cholangiocarcinoma cells. (E) The expression of miR-137 was inversely associated with that of WNT2B in cholangiocarcinoma tissues. (F) The mRNA expression levels of WNT2B were detected in 29 cholangiocarcinoma tissues and 20 adjacent tissues using reverse transcription-quantitative PCR. (G) The mRNA expression levels of WNT2B in the cholangiocarcinoma cell lines TFK-1, HuCCT1, RBE and QBC939 and HIBEpiCs were detected using reverse transcription-quantitative PCR. ${ }^{* *} \mathrm{P}<0.01$. WNT2B, Wnt family member $2 \mathrm{~B}$; UTR, untranslated region; HIBEpiCs, human intrahepatic biliary epithelial cells; NC, negative control; LV, lentivirus; miR, microRNA.

targeted genes of the Wnt signaling pathway, including c-Myc, $\mathrm{N}$-cadherin, vimentin, CDK2 and cyclin D1, were decreased in miR-137-overexpressing cells, whereas restoring the expression of WNT2B in miR-137-overexpressing cells reversed this effect (Fig. 6C and D). Taken together, these findings indicate that miR-137 may inhibit Wnt-related signaling pathways via WNT2B.

Restoration of $W N T 2 B$ expression reverses the inhibitory effects of miR-137. The present study investigated whether
WNT2B overexpression was able to reverse the inhibitory effects of miR-137 on CCA cells. First, the results of the CCK-8 assays indicated that restoration of WNT2B expression in cells stably expressing miR-137 reversed the inhibitory effect of miR-137 on cell proliferation (Fig. 7A). Similarly, Transwell assays revealed that restoring the expression level of WNT2B in miR-137-overexpressing cells relieved the inhibitory effect of miR-137 on cell mobility (Fig. 7B). Overall, these results demonstrated that restoration of WNT2B expression can reverse the inhibitory effects of miR-137 on CCA cells. 


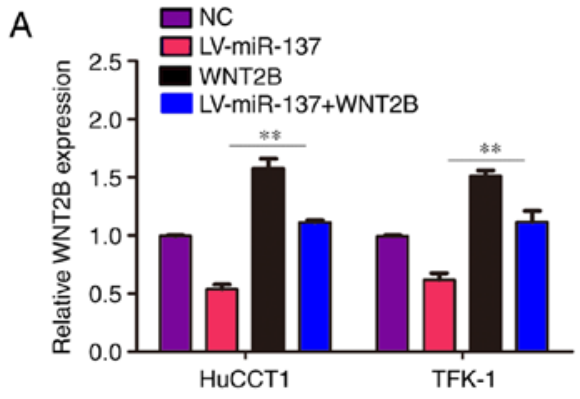

C

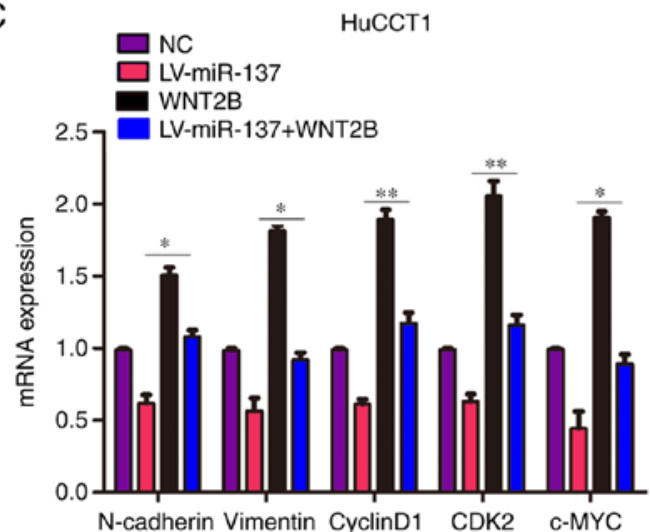

B

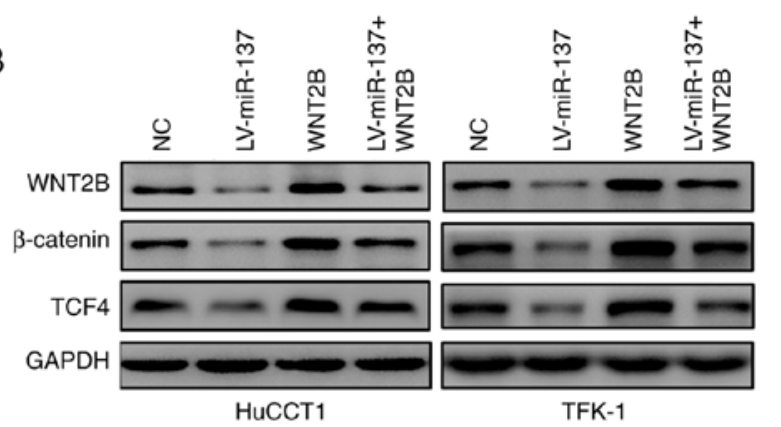

TFK-1

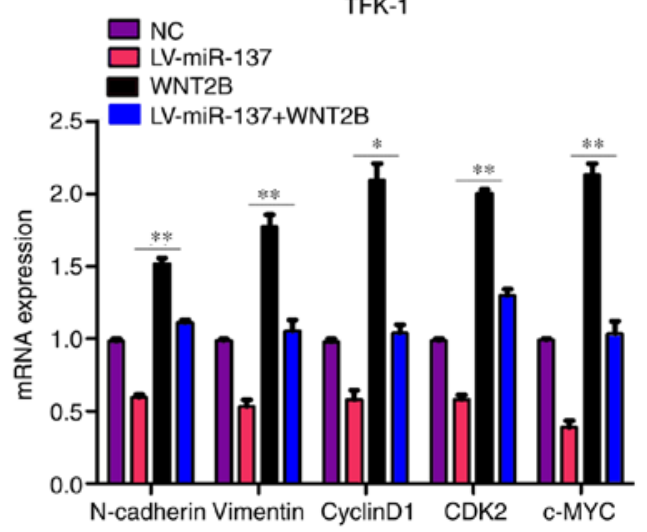

D
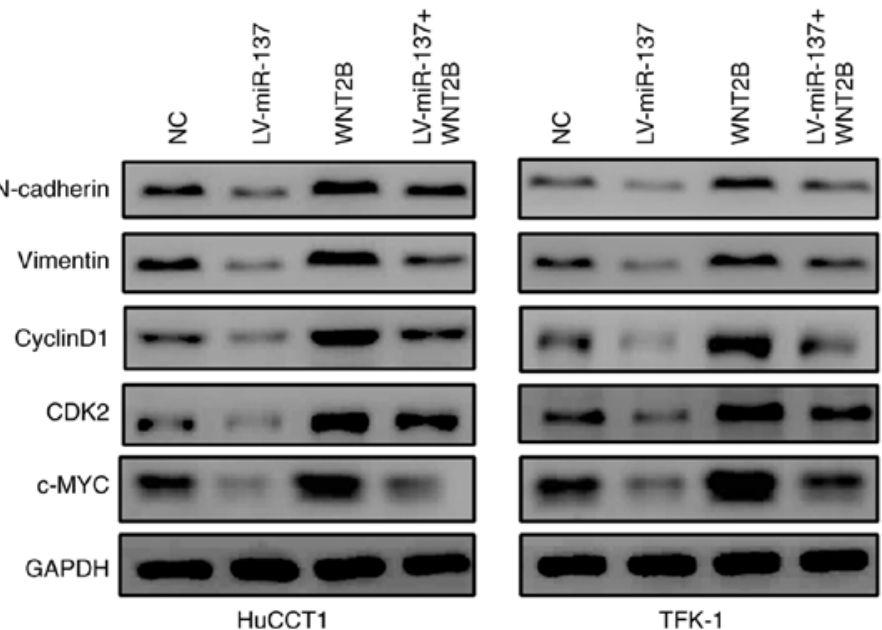

Figure 6. miR-137 regulates the Wnt signaling pathway via WNT2B. Cells were divided into four groups and subjected to different treatments: NC lentiviruses; transduction of miR-137 lentiviruses (LV-miR-137) alone; treatment with WNT2B plasmid (WNT2B) alone; transduction of miR-137 lentiviruses and treatment with WNT2B plasmid (LV-miR-137 + WNT2B). (A) The mRNA level of WNT2B in each group was detected using reverse transcription-quantitative PCR. (B) The protein expression levels of WNT2B, $\beta$-catenin and TCF4 in each group were detected using western blotting. (C) The mRNA levels of $\mathrm{N}$-cadherin, vimentin, cyclin D1, CDK2 and c-Myc in each group was detected using reverse transcription-quantitative PCR. (D) The protein levels of $\mathrm{N}$-cadherin, vimentin, cyclin $\mathrm{D} 1, \mathrm{CDK} 2$ and c-Myc in each group were detected using western blotting. ${ }^{*} \mathrm{P}<0.05,{ }^{* *} \mathrm{P}<0.01$. WNT2B, Wnt family member $2 \mathrm{~B}$; CDK, cyclin-dependent kinase; NC, negative control; LV, lentivirus; miR, microRNA; N, neural.

\section{Discussion}

Various studies have demonstrated that the miRNA class of non-coding RNAs participate in tumor progression through regulating a series of target genes involved in tumor proliferation and metastasis (24). Dysregulated expression or function of miRNAs has been demonstrated in a series of malignancies, including CCA (25). For example, miR-142-5p was shown to decrease the expression of phosphatase and tensin homolog and promote the progression of CCA (26). It was demonstrated that miR-329 can suppress the expression of pituitary tumor-transforming 1 , inactivates the mitogen-activated protein kinase/extracellular signal-regulated kinase signaling pathway and has the potential to suppress CCA cell proliferation (27). Even though numerous miRNAs may be involved in the development of CCA, the present study only focused on miR-137, exploring its role and its target gene, WNT2B, in CCA biology.

The present study verified that the expression of miR-137 was decreased in CCA tissues compared with that in adjacent non-tumor tissues. It was also indicated that the expression of miR-137 was markedly lower in CCA cell lines compared with that in HIBEpiCs and miR-137 overexpression inhibited CCA cell proliferation, decreased the ability of colony formation, 
A

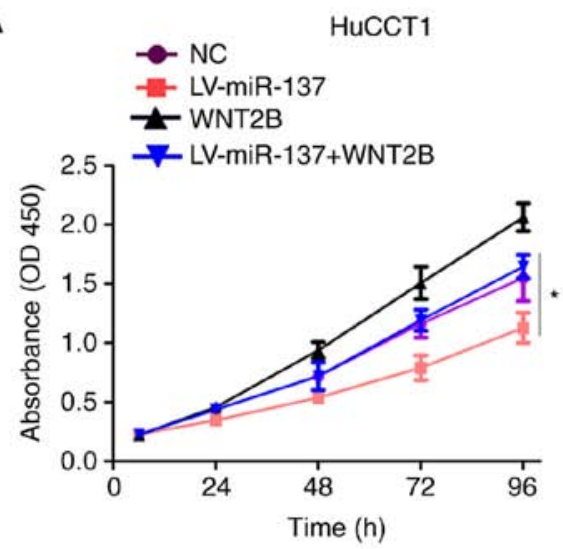

B
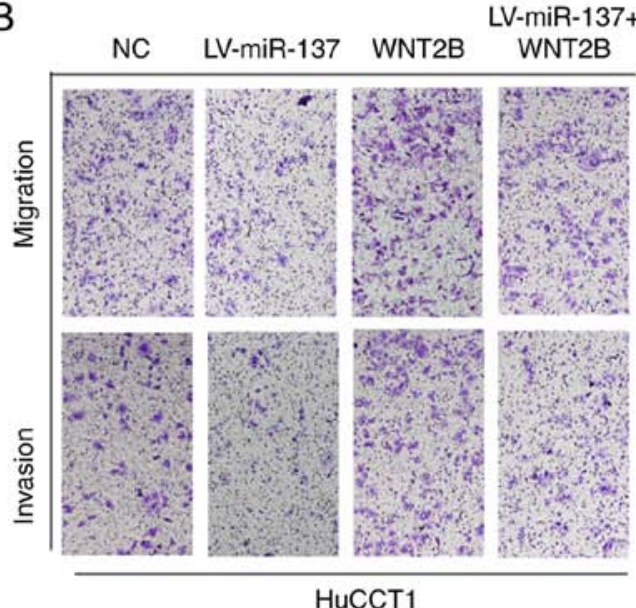

HuCCT1

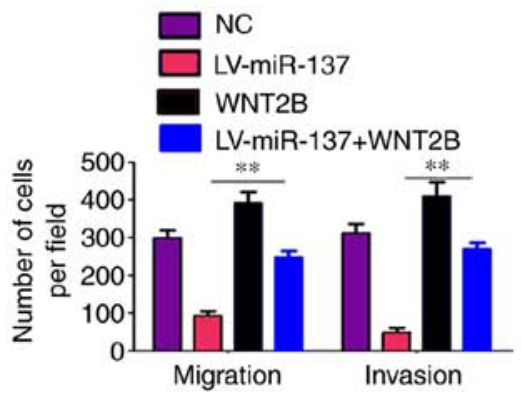

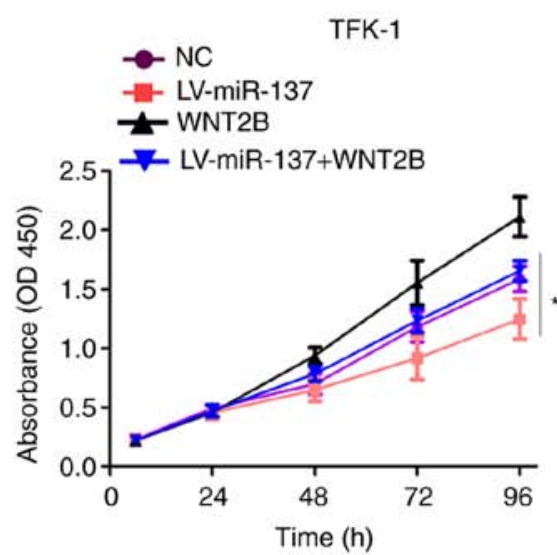
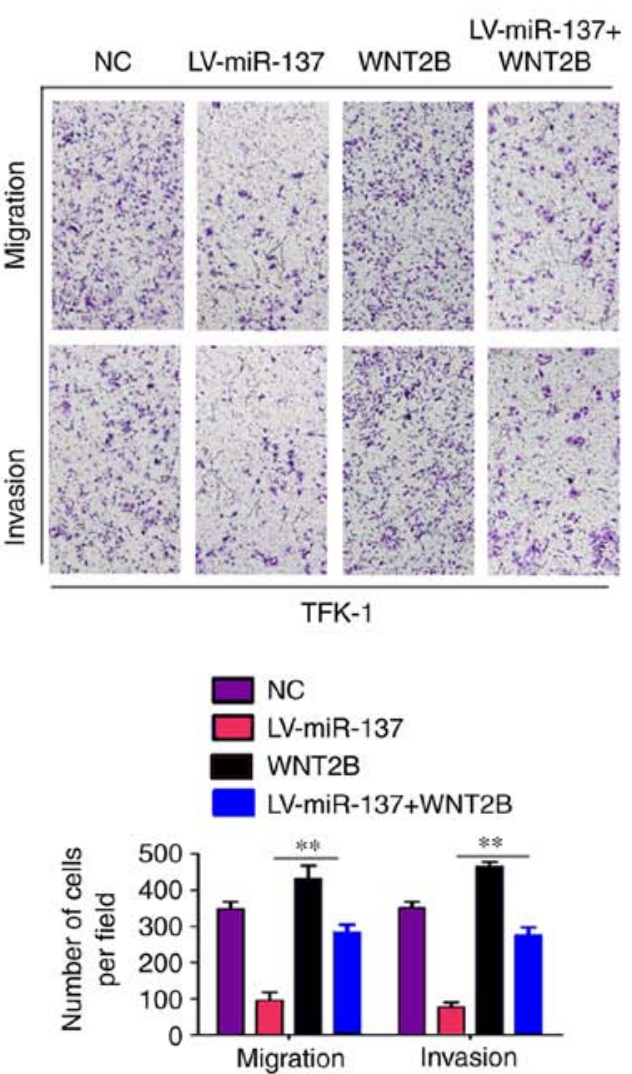

Figure 7. Restoration of WNT2B reverses the inhibitory effects of miR-137. Cells were divided into four groups and subjected to different treatments: NC lentiviruses (NC); transfection of miR-137 lentiviruses (LV-miR-137) alone; treatment with WNT2B plasmid (WNT2B) alone; transduction of miR-137 lentiviruses and treatment with WNT2B plasmid (LV-miR-137 + WNT2B). (A) Cell Counting Kit-8 assays were used to detect the proliferation ability of each group. (B) Transwell assays were used to measure the migration and invasion ability of each group. Magnification, $\mathrm{x} 40 .{ }^{*} \mathrm{P}<0.05$, ${ }^{* * *} \mathrm{P}<0.01$. WNT2B, Wnt family member 2B; NC, negative control; miR, microRNA.

induced cell cycle arrest, and inhibited cell migration and invasion. This constitutes evidence that miR-137 may act as a tumor suppressor in CCA.

WNT2B, also referred to as WNT13, is the thirteenth Wnt gene to be cloned. WNT2B interacts with frizzled receptor and activates the Wnt signaling pathway, which leads to the increased expression of $\beta$-catenin in the cytoplasm (23). Subsequently, the $\beta$-catenin protein translocates from the cytoplasm into the nucleus and promotes the transcriptional effect of TCF4 $(28,29)$. Previous studies have reported that WNT2B acts as an oncogene in a number of tumors and is positively associated with tumor progression. WNT2B increases cell proliferation ability and induces EMT via activating $\beta$-catenin-related pathways in non-small cell lung cancer (22). WNT2B inhibition was found to increase the chemosensitivity of head and neck squamous cell carcinoma (30). The expression of WNT2B was also found to be higher in pancreatic cancer tissues and high mRNA levels of WNT2B were positively associated with unfavorable prognosis in patients suffering from pancreatic cancer (31). To the best of our knowledge, the present study is the first to demonstrate that miR-137 targets WNT2B in CCA. The expression levels of WNT2B were negatively associated with miR-137 in CCA tissues. miR-137 decreased the expression of WNT2B and its downstream pathway molecules, while restoration of WNT2B expression alleviated the inhibitory effect of miR-137. Overall, 
the present study provided evidence that miR-137 has the potential to suppress CCA cell proliferation and mobility via decreasing WNT2B translation.

Therefore, miR-137 and WNT2B may prove to be useful diagnostic biomarkers as well as effective targets for the clinical treatment of CCA. Furthermore, the findings of the present study may improve understanding of the molecular mechanism underlying the development of CCA.

\section{Acknowledgements}

Not applicable.

\section{Funding}

The present study was supported by the National Natural Science Foundation of China (grant no. 81060176) and the Major Projects of Applied and Basic Research Program of Guizhou Province [grant no. J-(2015)2003].

\section{Availability of data and materials}

The datasets used and/or analyzed during the current study are available from the corresponding author on reasonable request.

\section{Authors' contributions}

SL ZZ, SP and JZ performed the experiments. YX, YS, JL, $\mathrm{SX}, \mathrm{DM}$ and BG were responsible for analysis and interpretation of the results. TC designed the experiments and wrote the manuscript. All the authors have read and approved the final version of the manuscript.

\section{Ethics approval and consent to participate}

The Human Trial Ethics Committee of Guizhou Medical University approved the study. Written informed consent was obtained from the patients who provided the specimens. The present study was performed in accordance with the principles outlined in the Declaration of Helsinki.

\section{Patient consent for publication}

Not applicable.

\section{Competing interests}

The authors declare that they have no competing interests.

\section{References}

1. Ma WJ, Wu ZR, Shrestha A, Yang Q, Hu HJ, Wang JK, Liu F, Zhou RX, Li QS and Li FY: Effectiveness of additional resection of the invasive cancer-positive proximal bile duct margin in cases of hilar cholangiocarcinoma. Hepatobiliary Surg Nutr 7: 251-269, 2018.

2. Liang W, Xu L, Yang P, Zhang L, Wan D, Huang Q, Niu T and Chen F: Novel nomogram for preoperative prediction of early recurrence in intrahepatic cholangiocarcinoma. Front Oncol 8: 360, 2018.

3. Beal EW, Tumin D, Moris D, Zhang XF, Chakedis J, Dilhoff M, Schmidt CM and Pawlik TM: Cohort contributions to trends in the incidence and mortality of intrahepatic cholangiocarcinoma. Hepatobiliary Surg Nutr 7: 270-276, 2018.
4. Rizvi S, Khan SA, Hallemeier CL, Kelley RK and Gores GJ: Cholangiocarcinoma-evolving concepts and therapeutic strategies. Nat Rev Clin Oncol 15: 95-111, 2018.

5. Burroughs AM and Ando Y: Identifying and characterizing functional 3 ' nucleotide addition in the miRNA pathway. Methods 152: 23-30, 2019.

6. Lee D and Shin C: MicroRNA-target interactions: New insights from genome-wide approaches. Ann N Y Acad Sci 1271: 118-128, 2012.

7. Puik JR, Meijer LL, Le Large TY, Prado MM, Frampton AE, Kazemier $\mathrm{G}$ and Giovannetti E: miRNA profiling for diagnosis, prognosis and stratification of cancer treatment in cholangiocarcinoma. Pharmacogenomics 18: 1343-1358, 2017.

8. Wan P, Chi X, Du Q, Luo J, Cui X, Dong K, Bing Y, Heres C and Geller DA: miR-383 promotes cholangiocarcinoma cell proliferation, migration, and invasion through targeting IRF1. J Cell Biochem 119: 9720-9729, 2018.

9. Xu Z, Liu G, Zhang M, Zhang Z, Jia Y, Peng L, Zhu Y, Hu J, Huang $R$ and Sun $X$ : miR-122-5p inhibits the proliferation, invasion and growth of bile duct carcinoma cells by targeting ALDOA. Cell Physiol Biochem 48: 2596-2606, 2018.

10. Ma J, Weng L, Wang Z, Jia Y, Liu B, Wu S, Cao Y, Sun X, Yin X, Shang $M$ and Mao A: MiR-124 induces autophagy-related cell death in cholangiocarcinoma cells through direct targeting of the EZH2-STAT3 signaling axis. Exp Cell Res 366: 103-113, 2018.

11. He Z, Guo X, Tian S, Zhu C, Chen S, Yu C, Jiang J and Sun C: MicroRNA-137 reduces stemness features of pancreatic cancer cells by targeting KLF12. J Exp Clin Cancer Res 38: 126, 2019.

12. Xiao J, Peng F, Yu C, Wang M, Li X, Li Z, Jiang J and Sun C: microRNA-137 modulates pancreatic cancer cells tumor growth, invasion and sensitivity to chemotherapy. Int J Clin Exp Pathol 7: 7442-7450, 2014.

13. Min L, Wang F, Hu S, Chen Y, Yang J, Liang S and Xu X: Aberrant microRNA-137 promoter methylation is associated with lymph node metastasis and poor clinical outcomes in non-small cell lung cancer. Oncol Lett 15: 7744-7750, 2018.

14. Wu QQ, Zheng B, Weng GB, Yang HM, Ren Y, Weng XJ, Zhang SW and Zhu WZ: Downregulated NOX4 underlies a novel inhibitory role of microRNA-137 in prostate cancer. J Cell Biochem 120: 10215-10227, 2019.

15. Zhao X, Lu C, Chu W, Zhang B, Zhen Q, Wang R, Zhang Y, Li Z, Lv B, Li H and Liu J: MicroRNA-124 suppresses proliferation and glycolysis in non-small cell lung cancer cells by targeting AKT-GLUT1/HKII. Tumour Biol 39: 1010428317706215, 2017.

16. Sakaguchi M, Hisamori S, Oshima N, Sato F, Shimono Y and Sakai Y: miR-137 regulates the tumorigenicity of colon cancer stem cells through the inhibition of DCLK1. Mol Cancer Res 14: 354-362, 2016.

17. Bi WP, Xia M and Wang XJ: miR-137 suppresses proliferation, migration and invasion of colon cancer cell lines by targeting TCF4. Oncol Lett 15: 8744-8748, 2018.

18. Livak KJ and Schmittgen TD: Analysis of relative gene expression data using real-time quantitative PCR and the 2(-Delta Delta C(T)) method. Methods 25: 402-408, 2001.

19. Zhang XY, Gao PT, Yang X, Cai JB, Ding GY, Zhu XD, Ji Y, Shi GM, Shen YH, Zhou J, et al: Reduced selenium-binding protein 1 correlates with a poor prognosis in intrahepatic cholangiocarcinoma and promotes the cell epithelial-mesenchymal transition. Am J Transl Res 10: 3567-3578, 2018.

20. Zou Z, Zheng B, Li J, Lv X, Zhang H, Yu F, Kong L, Li Y, Yu M, Fang L and Liang B: TPX2 level correlates with cholangiocarcinoma cell proliferation, apoptosis, and EMT. Biomed Pharmacother 107: 1286-1293, 2018.

21. Peng R, Zhang PF, Zhang C, Huang XY, Ding YB, Deng B, Bai DS and Xu YP: Elevated TRIM44 promotes intrahepatic cholangiocarcinoma progression by inducing cell EMT via MAPK signaling. Cancer Med 7: 796-808, 2018.

22. Wang B, Sun L, Li J and Jiang R: miR-577 suppresses cell proliferation and epithelial-mesenchymal transition by regulating the WNT2B mediated Wnt/ $\beta$-catenin pathway in non-small cell lung cancer. Mol Med Rep 18: 2753-2761, 2018.

23. Jiang Z, Jiang C and Fang J: Up-regulated lnc-SNHG1 contributes to osteosarcoma progression through sequestration of miR-577 and activation of WNT2B/Wnt/ $\beta$-catenin pathway. Biochem Biophys Res Commun 495: 238-245, 2018.

24. Plieskatt JL, Rinaldi G, Feng Y, Peng J, Yonglitthipagon P, Easley S, Laha T, Pairojkul C, Bhudhisawasdi V, Sripa B, et al: Distinct miRNA signatures associate with subtypes of cholangiocarcinoma from infection with the tumourigenic liver fluke Opisthorchis viverrini. J Hepatol 61: 850-858, 2014. 
25. Chen Y, Liu D, Liu P, Chen Y, Yu H and Zhang Q: Identification of biomarkers of intrahepatic cholangiocarcinoma via integrated analysis of mRNA and miRNA microarray data. Mol Med Rep 15: 1051-1056, 2017.

26. Wei G, Yuan Y, He X, Jin L and Jin D: Enhanced plasma miR-142-5p promotes the progression of intrahepatic cholangiocarcinoma via targeting PTEN. Exp Ther Med 17: 4190-4196, 2019.

27. Liang HQ, Wang RJ, Diao CF, Li JW, Su JL and Zhang S: The PTTG1-targeting miRNAs miR-329, miR-300, miR-381, and miR-655 inhibit pituitary tumor cell tumorigenesis and are involved in a p53/PTTG1 regulation feedback loop. Oncotarget 6: 29413-29427, 2015.

28. Zhu X, Yuan C, Tian C, Li C, Nie F, Song X, Zeng R, Wu D, Hao $X$ and Li L: The plant sesquiterpene lactone parthenolide inhibits Wnt/beta-catenin signaling by blocking synthesis of the transcriptional regulators TCF4/LEF1. J Biol Chem 293: $5335-5344,2018$
29. Wang $Y$, Lin $P$, Wang $Q$, Zheng $M$ and Pang L: Wnt3a-regulated TCF4/ $\beta$-catenin complex directly activates the key Hedgehog signalling genes Smo and Gli1. Exp Ther Med 16: 2101-2107, 2018.

30. Li SJ, Yang XN and Qian HY: Antitumor effects of WNT2B silencing in GLUT1 overexpressing cisplatin resistant head and neck squamous cell carcinoma. Am J Cancer Res 5: 300-308, 2014.

31. Jiang H, Li F, He C, Wang X, Li Q and Gao H: Expression of Gli1 and Wnt2B correlates with progression and clinical outcome of pancreatic cancer. Int J Clin Exp Pathol 7: 4531-4538, 2014.

This work is licensed under a Creative Commons Attribution-NonCommercial-NoDerivatives 4.0 International (CC BY-NC-ND 4.0) License. 